\title{
Using an agent-based model to analyze the dynamic communication network of the immune response
}

Virginia A Folcik ${ }^{1,3^{*}}$, Gordon Broderick², Shunmugam Mohan ${ }^{1}$, Brian Block ${ }^{1,3}$, Chirantan Ekbote ${ }^{1,3}$, John Doolittle ${ }^{1,3}$, Marc Khoury ${ }^{1,3}$, Luke Davis ${ }^{1}$, Clay B Marsh ${ }^{4,1}$

\footnotetext{
* Correspondence: nivar.1@osu.edu 1 Department of Internal Medicine, Division of Pulmonary, Allergy, Critical Care and Sleep Medicine, The Ohio State University Medical Center, Davis Heart and Lung Research Institute, Columbus, $\mathrm{OH}$, USA
}

\begin{abstract}
Background: The immune system behaves like a complex, dynamic network with interacting elements including leukocytes, cytokines, and chemokines. While the immune system is broadly distributed, leukocytes must communicate effectively to respond to a pathological challenge. The Basic Immune Simulator 2010 contains agents representing leukocytes and tissue cells, signals representing cytokines, chemokines, and pathogens, and virtual spaces representing organ tissue, lymphoid tissue, and blood. Agents interact dynamically in the compartments in response to infection of the virtual tissue. Agent behavior is imposed by logical rules derived from the scientific literature. The model captured the agent-to-agent contact history, and from this the network topology and the interactions resulting in successful versus failed viral clearance were identified. This model served to integrate existing knowledge and allowed us to examine the immune response from a novel perspective directed at exploiting complex dynamics, ultimately for the design of therapeutic interventions.
\end{abstract}

Results: Analyzing the evolution of agent-agent interactions at incremental time points from identical initial conditions revealed novel features of immune communication associated with successful and failed outcomes. There were fewer contacts between agents for simulations ending in viral elimination (win) versus persistent infection (loss), due to the removal of infected agents. However, early cellular interactions preceded successful clearance of infection. Specifically, more Dendritic Agent interactions with TCell and BCell Agents, and more BCell Agent interactions with TCell Agents early in the simulation were associated with the immune win outcome. The Dendritic Agents greatly influenced the outcome, confirming them as hub agents of the immune network. In addition, unexpectedly high frequencies of Dendritic Agent-self interactions occurred in the lymphoid compartment late in the loss outcomes.

Conclusions: An agent-based model capturing several key aspects of complex system dynamics was used to study the emergent properties of the immune response to viral infection. Specific patterns of interactions between leukocyte agents occurring early in the response significantly improved outcome. More interactions at later stages correlated with persistent inflammation and infection. These simulation experiments highlight the importance of commonly overlooked aspects of the immune response and provide insight into these processes at a resolution level exceeding the capabilities of current laboratory technologies.

\section{Biomed Central}




\section{Background}

The immune system is a dynamic network of interacting cells that communicate directly and indirectly to exchange information during an immune response. Orosz gave this complex phenomenon the name "Immuno-ecology" [1], and described in detail the properties of the immune network, likening the immune response to swarming ants. The network qualities exhibited by the immune system allow such a geographically dispersed glandular system to effectively maintain homeostasis and yet swiftly react in a de novo, swarm-like manner when responding to a pathogen. Immune cell activity is controlled by cell-cell interaction and by environmental signals that these and other cells produce. These signals constitute broadcast signals if they enter the blood. In contrast, cell-to-cell interactions constitute direct communication. The combination of indirect and direct communication with connections changing over time gives the immune system its network topology. The immuno-ecology view of the immune network identifies immune cells as nodes and cytokines and chemokines as edges or links between the nodes. This network topology evolves over time as cells interact, change state and eventually die. An immune response most effectively protects the body when the leukocytes rapidly eliminate pathogens and then naturally diminish in numbers (via apoptosis), avoiding damaging chronic inflammation [2-5].

In real world networks such as the world-wide web [6] and the biochemistry of living organisms [7], some nodes play a more central role than others. This network topology is called "scale-free", and is characterized by many nodes having very few links and a few "hub" nodes having many links [6]. In these cases the distribution of connections among the network nodes follows a "power-law". This hub-centric architectural design provides a high level of resilience to random loss of connections, yet makes these networks susceptible to attacks directed specifically at the hubs [8]. This scale-free topology was demonstrated in simulation experiments conducted with the Basic Immune Simulator (BIS) and has been reported previously [9].

Others have also studied the network properties of the immune system [10-12] using a growing body of biochemically validated information describing cellular signaling pathways. Fuite, Vernon and Broderick [13] extended this elemental approach by identifying signaling networks using data from high-throughput molecular assays used to survey immune and neuroendocrine status. They applied novel topological analyses to identify network features that distinguished patients with chronic fatigue syndrome (CFS) from non-fatigued subjects. In a complex illness like CFS, the identification of individual biomarkers in human data is especially difficult because of the natural heterogeneity in the magnitude of cytokines and hormones normally produced [1]. Importantly, analyzing co-expression networks improved resolution and added a new dimension to molecular phenotyping [13]. Moreover, novel therapeutic strategies could prevent or enhance indirect and direct interactions between immune cells that are causing pathological inflammation or undesired immunosuppression [1].

In these examples, the immune networks were constructed with nodes representing immune cell types and the links between the nodes represented soluble mediators such as cytokines, chemokines, or hormones. Cell-cell signals mediated by direct contact were implicitly represented. In some cases, mediators of indirect communication or stigmergy $[14,15]$ were represented explicitly as nodes. Though revealing, these are 
typically static representations of network interactions and describe an average state of network assembly. The dynamic, spontaneous assembly and disassembly of network components that occurs over time were not described.

This study uses an agent-based model to explore the dynamics of immune network connectivity in cellular communication by direct cell-cell contact. In the static representation of the network model, discrete agents representing individual immune cells define the nodes (Figure 1). Connections between any two nodes involve direct physical contact leading to information exchange between individual immune cell agents. The agents and signals representing the various cell types and cytokines are described in additional file 1. A key advantage of using an agent-based model like the BIS_2010 (the current version of the BIS) is that it integrates experimental results from a wide range of studies, compiling them into a detailed set of known and validated interaction rules (additional file 2; [16]), and using the knowledge base in a way that allows observation and analysis of virtual cellular behavior. This agent-based approach allows a dynamic analysis of leukocyte interactions during an immune response to challenge. Though fluorescent leukocyte tagging in vivo continues to advance as a technology for studying cellular interaction, it is not possible to conduct analyses of immune dynamics experimentally at this level of detail and breadth, making simulation experiments highly useful.

Using this model-based approach, we identified patterns of temporally distinct network interactions that emerged from the contacts between individual agents during inflammation that led to different immunological win and loss outcomes [16]. By

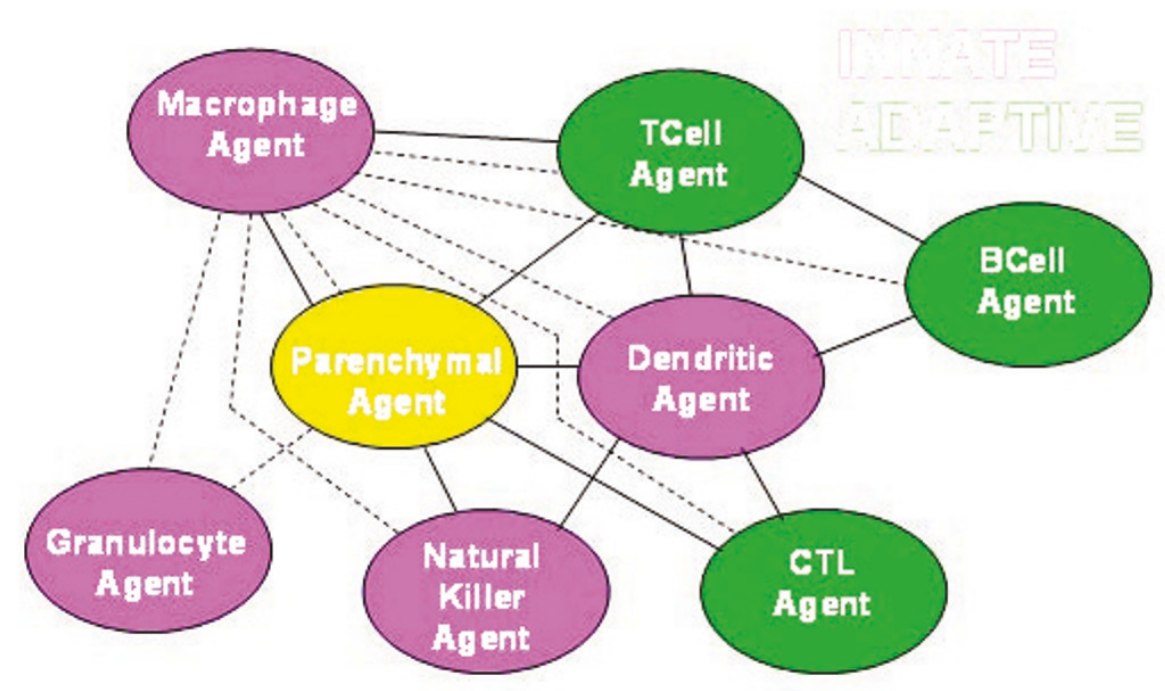

Figure 1 The BIS_2010 agents representing nodes in a static representation of the immune network. Each node in the immune network represents a category of immune cells that includes subtypes. Solid lines indicate two-way connections that involve a change in information recorded by both nodes upon contact. Dashed lines indicate interactions in which only one node, usually the Macrophage Agent, records information about the contact because the other node represents an agent that is dead. The agents representing leukocytes are pink or green, indicating their function in innate or adaptive immunity. 


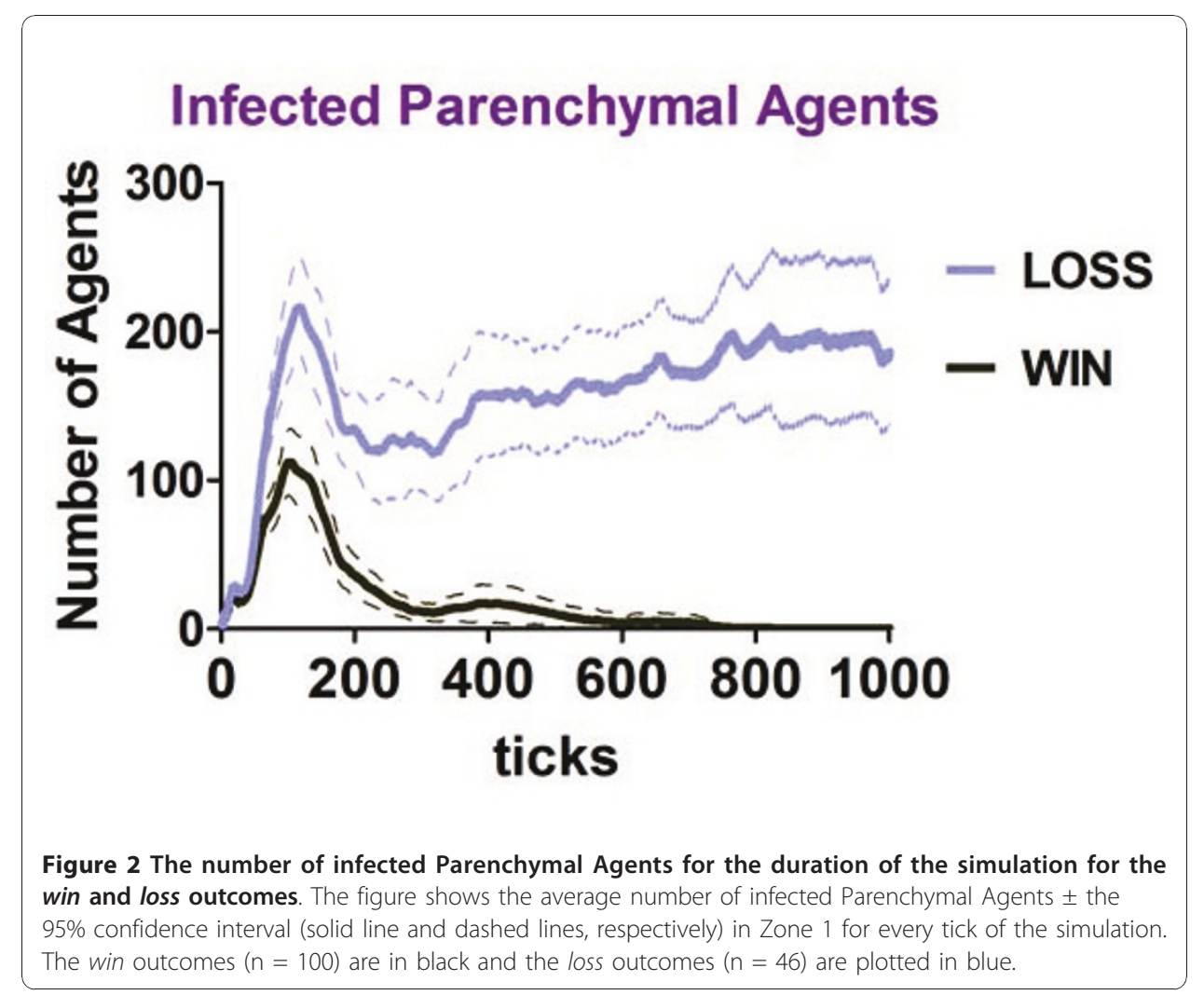

definition, a win occurred when the virtual infection of Parenchymal Agents was cleared and more than half of these agents survived or regenerated, a loss outcome occurred when the infection persisted in Parenchymal Agents (Figure 2), or they all died. The network interactions were also analyzed to identify characteristic features including interaction frequencies, the percent engagement of agents, and agent populations constituting functional hubs.

\section{Implementation}

The Basic Immune Simulator 2010 (BIS_2010)

The BIS and the new version, BIS_2010, were created using Repast] [17] in Java. Its purpose is to examine the activity of the immune system during an immune response to various pathogens and injury [16]. It is an agent-based model of the immune system with representations of the cells as agents (additional file 1), these agents have specified behaviors (additional file 2), and the tissue spaces where cellular interactions take place are represented as zones (additional file 3). The adjustable parameters and their initial values are provided in additional file 4 . The agents and spaces are extensions of Java classes in the RepastJ software library. The behavioral rules for the agents are described in detail in state diagrams (additional files 5, 6, 7, 8, 9, 10, 11, 12, 13, 14, 15, 16, 17, 18, $19,20,21$, and 22). The citations for empirical demonstration of immune cell behavior are in these state diagrams describing the rules. Time is represented as discrete, sequential "ticks" that allow agent behavioral events to emulate concurrency. Space and time in the model are abstractly represented. Though duration is not strictly represented, the correct sequence of events emerges from the behavioral rules of the agents, thereby providing an event-driven chronology. 
More specifically, at every tick each agent in the simulation is allowed to examine its immediate environment for signals or other agents. Each agent may react to what it detects, depending upon the rules that apply for the agent in its current state. It will only react if it detects a signal or agent relevant to its current state, and it can only change by one state, i.e. follow one edge to another state (per tick). Otherwise, it will remain in its current state until the next tick. Because many of the state changes represent behavioral events that occur within a solid tissue (as opposed to the blood), the exact quantity of time they require is unknown. Conditional control of events forces them to occur in the correct order.

One could estimate the quantity of time represented by ticks based upon the known duration of immunological events in human systems. The virus and the tissue are generic in the model and the space was based on human scale (described below), so hallmarks of the human immune response involving interactions of innate and adaptive immunity were used to estimate the time scale. The hallmarks used were the peaks of $\operatorname{IgM}$ and IgG antibody detection in the serum $[18,19]$, and the peaks of virus, IgM, and IgA detection at a mucosal surface [20]. The BIS_2010 correlates were the peaks of signals Ab5 (IgM), Ab1 and Ab2 (averaged; IgG) in Zone 3 (the blood); and the peaks of the signals for Virus, Ab5, Ab1 and Ab2 (averaged; IgA) in Zone 1 (the functional tissue space), respectively. An example calculation used the peak of detection of IgM in the serum, occurring at 7-10 days [18,19]. The peak of Ab5 in Zone 3 occurred at an average of 159 ticks (simulation time increments) for the win outcomes (data not shown). Using 8.5 days (the average of 7 and 10 days), 159 ticks/8.5 days is 18.7 ticks/day. There are 1440 minutes/day, and (1440 minutes/day)/(18.7 ticks/day) is 77 minutes/tick, or 1.3 hours/tick. This calculation was performed using six sets of input values from above (all obtained from win outcomes), with two different values for the day of peak IgG detection $[18,19]$. The average value obtained was 64 minutes/tick (range 45-86 minutes/tick) or approximately 1 hour/tick. If this value is applied to Figure 2, the peak of infected Parenchymal Agents occurs at 4.3 days for the win outcome.

Space was divided into discrete compartments where relative area in the BIS_2010 Zones approximates the volume of functional human tissue (Zone 1; a representative organ, such as the lungs), the secondary lymphoid tissue (Zone 2; a group of lymph nodes and spleen), and blood (Zone 3). The volume of the lungs in an adult is estimated to be $843 \pm 110 \mathrm{ml}$ [21], the volume of the lymph nodes in the thorax is approximately $12 \mathrm{ml}$ [22,23], and the spleen volume ranges from 180-250 $\mathrm{ml}$ [18]. The volume of blood in a human is approximately $5000 \mathrm{ml}$. The ratios of these volumes, roughly 1000:200:5000, were used to adjust the areas (number of $[x, y]$ coordinates in the square) of Zones 1, 2, and 3 to 12321:2500:62500, respectively.

\section{Simulation Runs and Initial Conditions}

A simulation run begins with all of the zones containing the numbers of agents specified in the initial conditions (additional file 4) randomly arranged (Zones 2 and 3) in whole or in part (Zone 1; additional file 3). When the BIS was first described, the initial parameters controlling the numbers of agents of different types were systematically varied and the outcomes compared [16]. Based on prior simulation runs and a parameter sweep of the number of Dendritic Agents, a (biologically) near-optimal set of experimental conditions were chosen from those producing the results shown in 
additional file 23 to examine the dynamics of immune network direct communication. Near-optimal was defined as the initial parameter values that resulted in a combination of a near maximal percentage of outcomes as wins yet enough losses to make comparisons of the win vs. loss data. The initial conditions chosen consisted of 200 Dendritic Agents and the other parameter values given in additional File 4. All of the data shown in Figures 2, 3, 4, 5, 6, 7, and 8, and additional files 24, 25, 26, 27, 28, and 29 came from 146 simulation runs with those initial conditions, resulting in 100 win outcomes and 46 loss outcomes for comparison.
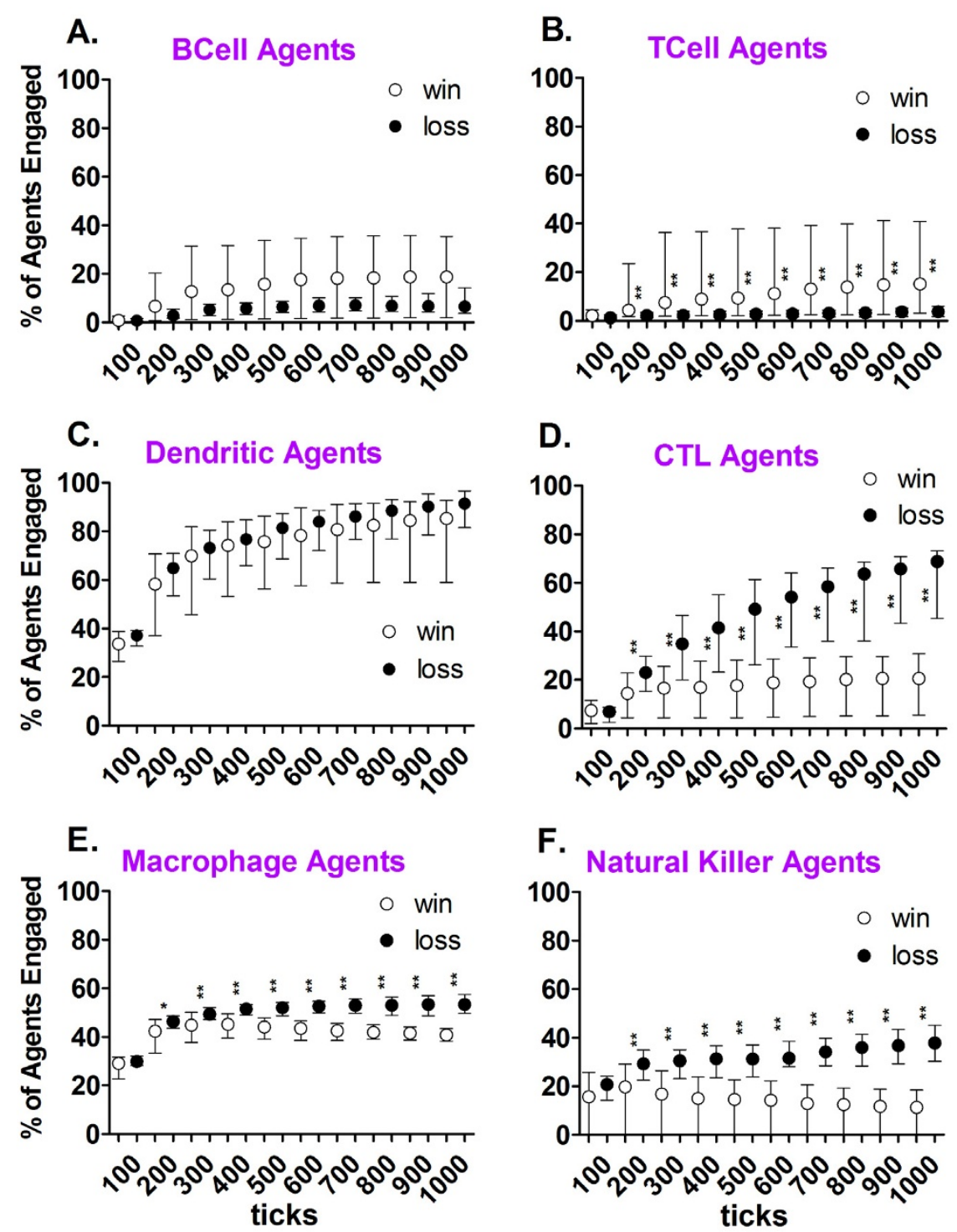

Figure 3 Percentage engagement in the virtual viral immune response for each immune agent type. The percentage of the each of the agents present in the simulation runs, in the win $(n=100)$ and the loss $(n=46)$ outcomes, that made a meaningful contact with at least one other agent were recorded cumulatively every 100 ticks. The data are expressed as the median percentage (circle) with the error bars showing the 25th and 75th percentiles. Asterisks between the win and loss results indicate significant differences at the recorded time point $\left({ }^{* *} \mathrm{p}\right.$-value $<=0.006$; ${ }^{*} \mathrm{p}$-value $\left.<=0.012\right)$ using a two-tailed MannWhitney U-test with the Bonferroni correction for multiple comparisons. 

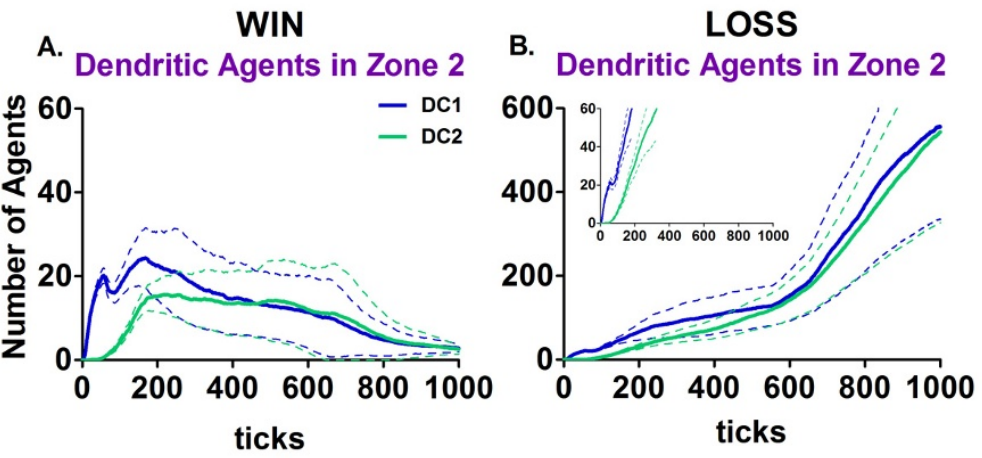

Figure 4 The number of activated Dendritic Agents (DCs) in Zone 2. A. The average number of proinflammatory Dendritic Agents (DC1; blue) and alternatively activated Dendritic Agents (DC2; green) \pm the 95\% confidence interval (solid line and dashed lines, respectively) for the win outcomes $(n=100)$. B. The average number of DC1 and DC2 \pm the 95\% confidence interval (solid line and dashed lines, respectively) for the loss outcomes $(n=46)$. The inset plot shows the loss data on the same scale as the win data in part A, for comparison.

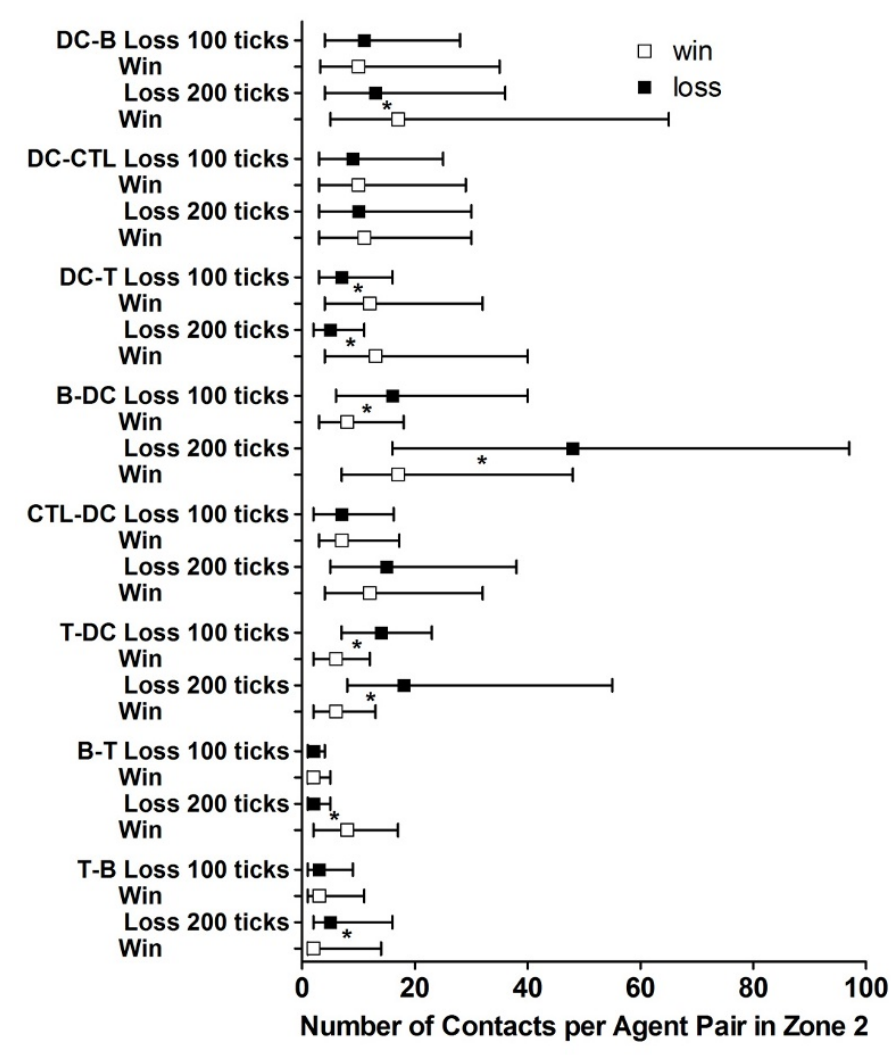

Figure $\mathbf{5}$ Quantities of specific interactions between agents representing leukocytes in Zone $\mathbf{2}$. The median (squares), $25^{\text {th }}$ percentile and $75^{\text {th }}$ percentile of number of links per node for the indicated combinations of agents and time points for the win ( $n=100$, open squares) and the loss ( $n=46$, filled squares) outcomes are shown. The first agent type listed indicates which agent recorded the contact. An asterisk between the win and loss results indicate significant differences at the recorded time point $\left({ }^{*}\right.$ p-value $<=$ 0.0016) using a two-tailed Mann-Whitney U-test with the Bonferroni correction for multiple comparisons. The abbreviations are as follows: B, BCell Agent; CTL, CTL Agent; DC, Dendritic Agent; T, TCell Agent. 


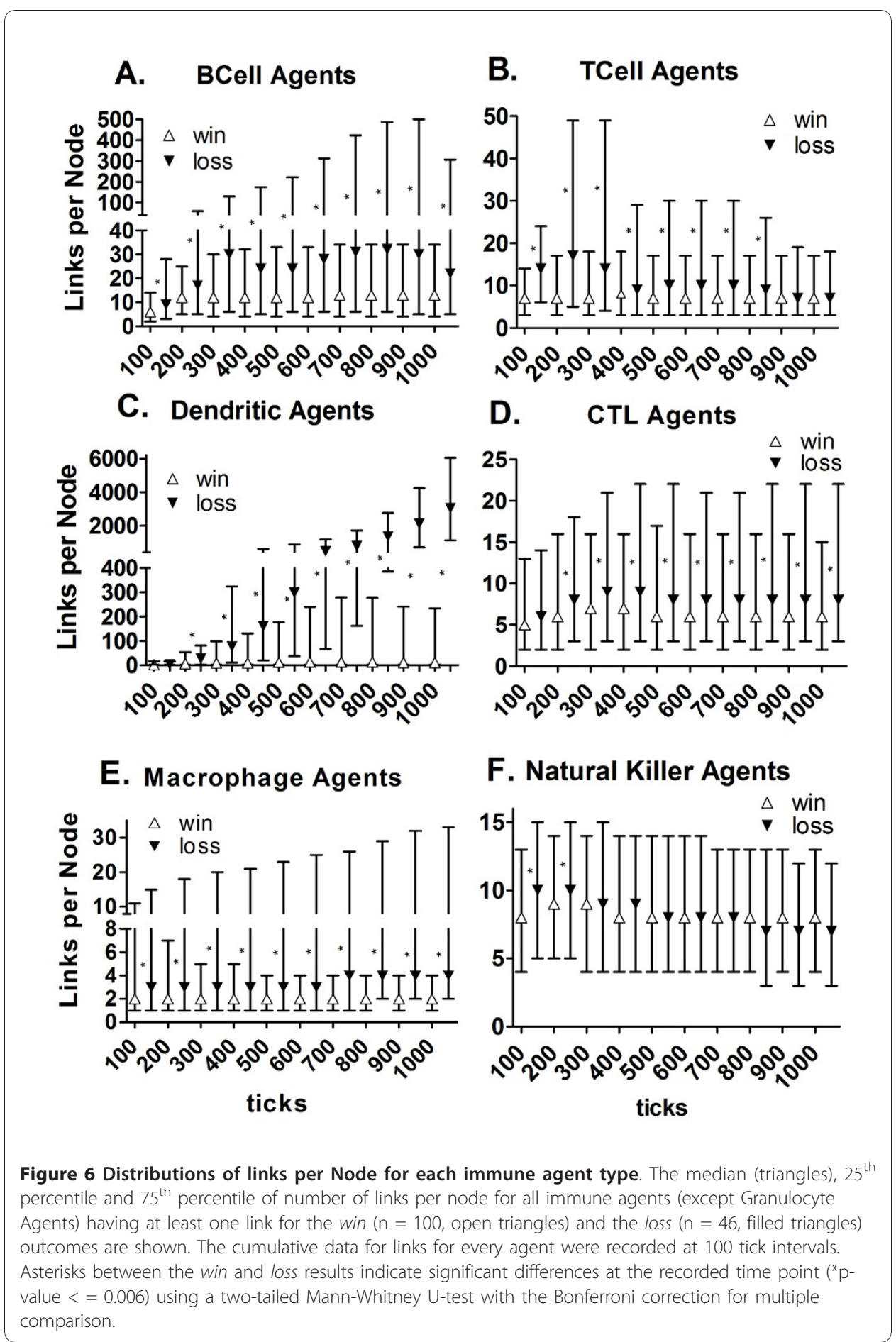

Agent Placement and Movement

The Parenchymal Agents representing the functional tissue cells (additional file 6) and the Portal Agents representing the entry and exit points for blood and lymphatic fluid (additional file 22) were placed in Zone 1 in the same pattern for every simulation run. Neither of these agent types moved in the tissue zone for the duration of the simulation, but they could die and be replaced depending upon the environmental conditions. 


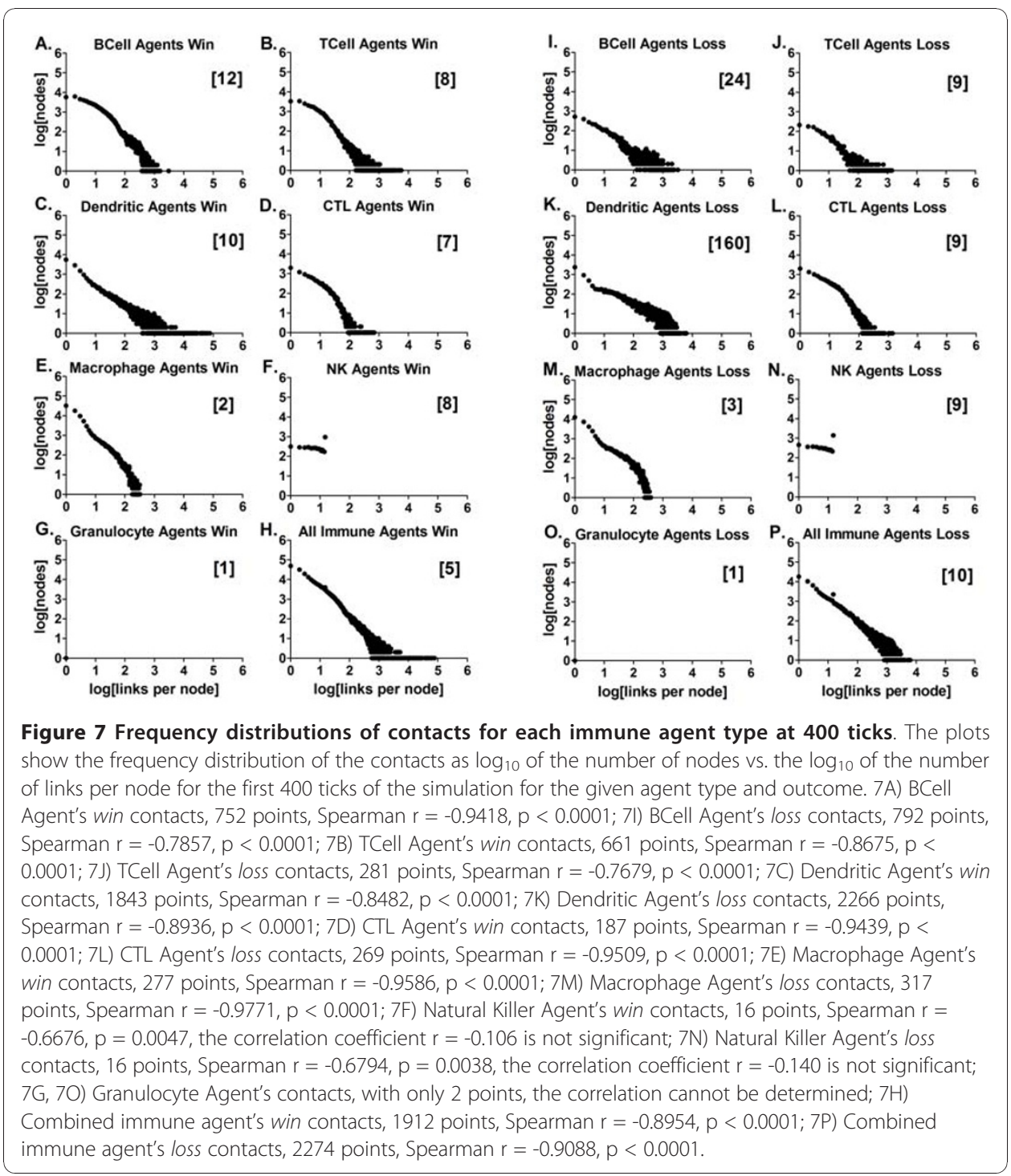

The conditions for replacement included the presence of surrounding uninfected Parenchymal Agents. The same four locations (coordinates of Parenchymal Agents) were chosen for the initial sites of viral infection for all data shown. Virus and PK1 signals (additional file 1) were produced at the first tick of the simulation, and after that point every simulation run produced a unique pattern.

Randomness is an integral part of the model, and is included as described here. All random numbers were generated from a uniform distribution. The Dendritic Agents, Macrophage Agents, Granulocyte Agents, and TCell, BCell and CTL Agents were placed randomly in their zones. The few lymphocyte agents specific for the virus (and the other scenarios) were placed at randomly chosen empty coordinates among non-specific lymphocyte agents in Zone 2. Agents moved randomly unless they were attracted by signals representing chemokines. Agent movement was in increments of one $[x, y]$ coordinate per tick. All signals diffused at each tick, using the Repast) "diffuse" method [17], which forms concentration gradients. Random initial placement (within the appropriate zones) and random movement of the agents or non-random movement towards chemotactic 


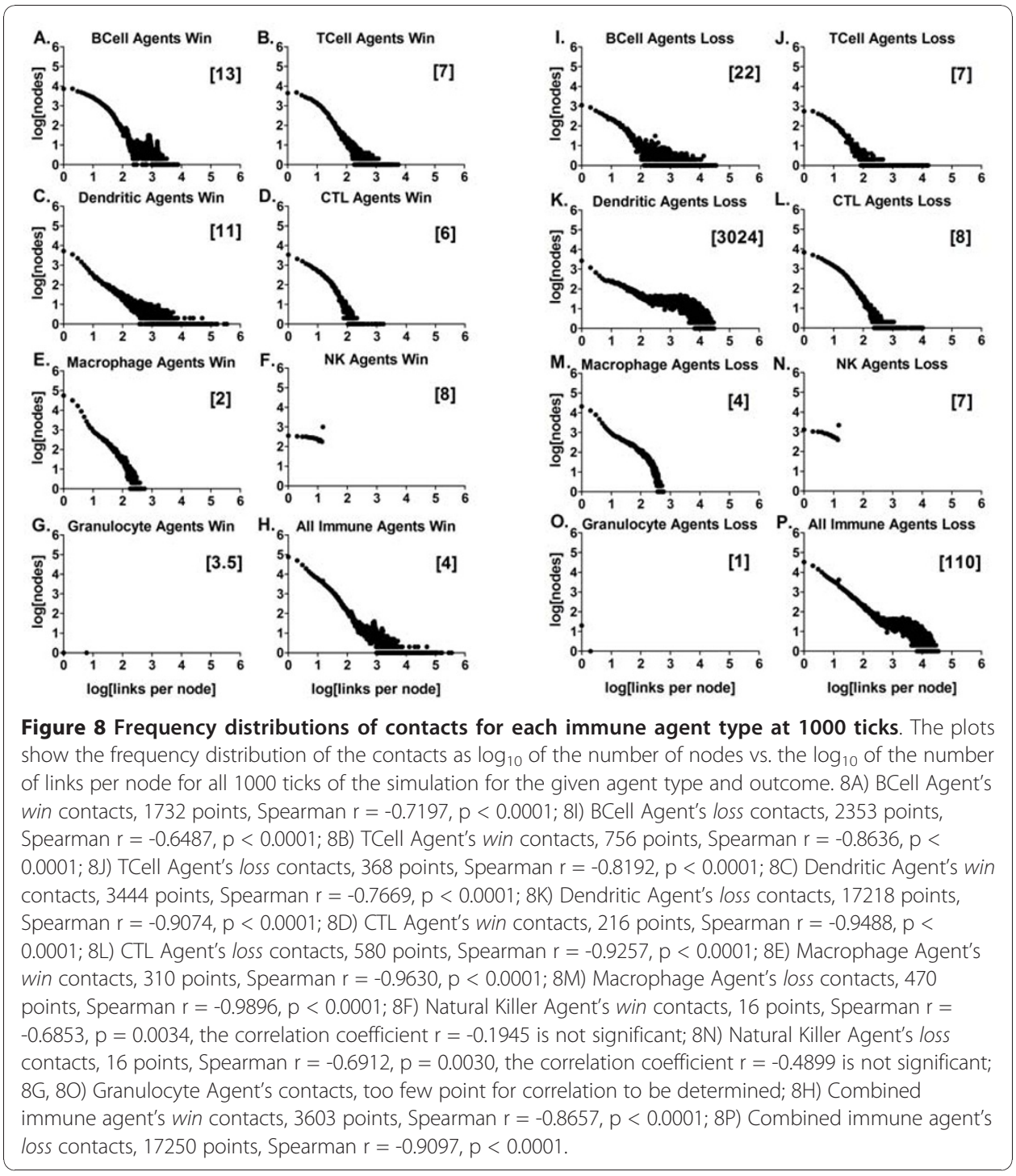

concentration gradients produced by other agents generated the observed patterns. This is an accurate representation of the immune system, because the cells of the immune system are initially distributed at random in the lymphoid tissue (in the naive individual) and migrate in response to environmental cues to carry out their functions [24]. Sometimes they follow biochemical gradients (chemotaxis), and sometimes they are subject to flow forces or cellular interactions in the lymphatics and in the circulation $[25,26]$ that may randomly change their arrival time at a new destination.

The lymphatic fluid ducts and blood vessels are represented by Portal Agents (additional file 22). When an agent leaves one zone via a Portal Agent it enters another zone at the site of a Portal Agent, under the control of environmental conditional rules at the entry site. If none of the Portal Agents in the new zone satisfy the conditions for entry (such as having the necessary chemotactic signals present proximally), the agent remains stationary and waits in a queue for the next tick. In this way, Portal Agents control the movement of agents and signals between zones. The agents or signals must be in the Portal Agent's Moore neighborhood (within the eight adjacent 
coordinate spaces to the Portal Agent) for this to occur. They might be considered to represent endothelial cells, which have been modeled by others for their contribution to systemic inflammation [27-29], but they are abstractly represented in the BIS_2010.

Updates included in BIS_2010

The BIS_2010 is an updated version of the agent-based model, the BIS, that was created using RepastJ $[17,30]$ and was previously described [16]. Because of the discovery and characterization of new types of T-helper lymphocytes including the T-helper $17 \mathrm{~s}$ [31-34], regulatory $\mathrm{T}$ cells (T-regs; [35-39]), and the T-follicular helper cells [40,41], the BIS_2010 was updated and these subtypes were added to the TCell Agent class (additional files 16, 17, and 18). Other additions included enhanced Macrophage Agent behavior (additional files 10,11, and 12) and Granulocyte Agent behavior (additional file 21) in response to other agents in apoptotic (non- inflammatory or programmed cell death) and necrotic (inflammatory; killed by environmental factors) states. BCell Agents were updated to include more behavioral states and antibody signals (additional files 13,14, and 15). The state diagrams for of all of the agents contain the details of their behavioral rules with literature citations, representing them as finite state automata. Agent behaviors are listed, categorized, and referenced in additional file 2. The list of references cited in the additional files is in additional file 30. Other updates to the BIS_2010 include the changes in the Zone areas described above.

\section{Code Verification}

When changes were made to the simulation program, the code for the agents' behavior was tested to ensure that it was executing correctly before the BIS_2010 was used for experiments. Verifying the code for the behavior of the agents in the BIS_2010 is challenging because it is a program with sections of code that execute stochastically. Besides the traditional methods for verification [42], including unit testing, code walkthroughs, and observation of the visual output (additional file 3) with input parameters set to produce expected patterns, we have created a program to automate tracing of agent behavior called the AgentVerifier [43], a separate application from the BIS_2010. This is a Java application that checks state transitions for the agents and any accompanying changes in internal variable values. This process was previously done by manually reading the BIS agent behavior output files [16].

\section{Recording the Dynamic Network Interactions}

The simulation was run and the interactions were recorded by having each agent count its contacts with other agents that either caused one of the agents involved to change state or one of the agents to change a value in an internal variable. These criteria defined "meaningful" contacts. Contacts between two agents that did not meet either of these criteria (random collisions) were not counted. Agents had to be within one coordinate space of each other (within the Moore neighborhood, radius =1), except for Dendritic Agents, which were allowed to probe a radius of two coordinate spaces (Moore neighborhood, radius $=2$ ). This represents the relatively large size of dendritic cells with their long dendrites [44,45]. In addition, multiple contacts between the same two agents were counted (at sequential ticks), as long as they remained in a state that 
recognized the contact (for example, one did not die). Thus, the BIS_2010 models processes that have been observed and recorded in living lymphoid tissue [44-53].

Each individual agent kept an ongoing record (a list of integer arrays) of their total number of meaningful contacts, including the agent types involved and the zone where the interactions took place. Both agents involved in an interaction recorded the interaction unless one of the agents was dead. Because Portal Agents represented structures and not individual cells, contacts were not recorded for these agents. Contact summaries were saved in text files with comma separated values at 100 tick intervals during each simulation run.

Signals are another major element in the BIS_2010. The signals represent cytokines and chemokines, biologically active proteins that direct migration and mediate information exchange. All of the signals that the agents produce are listed in additional file 1. Cytokines and chemokines drive cell-cell interaction by providing indirect communication or "stigmergy" [15], and have been considered to form a network of communication among the cells of the immune system [1,10,11,13]. Signals in the BIS_2010 control agent behavior by causing state transitions. The state of an agent determines whether the agent recognizes a contact with another agent or a signal, simulating the presence or absence of surface receptors on cells. Production of a signal may be common to multiple agent types, as described in additional file 1. For efficiency of execution, the BIS_2010 was not implemented in a manner that allows determination of the agent source of a signal. The impact of signals on network formation was implicitly captured in the network of direct communication events between agents.

\section{Statistical Analyses}

Non-parametric statistical methods were used unless otherwise indicated. A very conservative Bonferroni correction was applied to correct the alpha value used when estimating significance in multiple comparisons. Thus the alpha value necessary for significance was made smaller by dividing it by the number of comparisons made for a data set. Outcomes at regular time intervals were analyzed using separate statistical comparisons to simplify interpretation. GraphPad Prism version 5.03 was used to create the plots in the figures and perform the statistical analyses.

\section{Results}

\section{Simulation outcomes}

The initial conditions for the simulation runs used for the network analysis were chosen (from those shown in additional file 23) to provide mostly immune win outcomes but enough loss outcomes for comparisons to be made. In the win outcomes, all of the infected Parenchymal Agents were eliminated, usually within the first half of the simulation run (Figure 2). In the loss outcomes, more Parenchymal Agents became infected by the time 100 ticks had passed, and the virtual immune response failed to eliminate all of the infected agents. The data shown in the remainder of the figures came from the same simulation runs as the data shown in Figure 2. There were many ways to present the data derived from these experiments; the results are presented from an immunologist's perspective. 


\section{Participation of agents in mounting a typical immune response}

A characteristic of the immune response demonstrated by the BIS_2010 was that most of the agent types representing leukocytes never made meaningful contacts with any other agents (Figure 3). Agents having had at least one meaningful contact (in any Zone) were considered "engaged" in the immune response, and the extent of engagement of each agent type for the response duration was determined. Zero percent of the agents were engaged at the start of a simulation run. The median percent engaged and inter-quartile ranges are plotted in Figure 3 (with 100 tick intervals) and show the cumulative history of engagement of each agent type for the duration of the simulation runs. The levels of engagement for simulation runs that ended in win outcomes are separated from the same data for loss outcomes. The results show that with the exception of the Dendritic and BCell Agents (Figure 3C and 3A), the median percent of agents engaged from the win and loss outcomes diverged significantly at 200 ticks into the simulation.

The Dendritic Agents displayed the greatest increases in engagement at the beginning (100 and 200 ticks) of the virtual immune response (Figure 3C). This initial surge corresponds to the recognition of antigen by contact with infected Parenchymal Agents in Zone 1 (Figure 2; additional file 7). The Dendritic Agents are activated by contact with the infected Parenchymal Agents and migrate to Zone 2 (Figure 4; additional file 8). Figure 4 shows the average numbers of Dendritic Agents of two different phenotypes, $\mathrm{DC} 1 \mathrm{~s}$ representing the pro-inflammatory type and DC2 $\mathrm{s}$ the alternatively activated type [54-62]. Note the ten-fold difference in the scale for the loss outcomes, in part B, and the inset in part B for comparison to part A. The numbers of activated Dendritic Agents that travel to Zone 2 (lymph node equivalent) decreases after the infected Parenchymal Agents have been nearly eliminated (Figure 2) between 200 and 300 ticks for the win outcome (part A), but not in the loss outcome (part B). In Zone 2 the Dendritic Agents seek BCell, TCell and CTL Agents to make contact and present antigen (Figure 5). The lag in engaging these lymphocytic agent types (Figure 3A, B, and 3D) is due to the time required by the Dendritic Agents to encounter the few viral antigen-matched lymphocyte agents in Zone $2[26,46,63]$. The requirement for viral antigen-specificity also accounts for the low level of lymphocyte agent engagement, especially apparent for the BCell Agents and TCell Agents in Figure 3.

The Natural Killer (NK) Agents and Macrophage Agents (Figure 3E and 3F) represent cells involved in the innate immune response (additional files 9, 10, 11, and 12). A greater percentage of these agents became engaged in the loss than in the win outcome. This is because the NK and Macrophage Agents continued to be engaged by infected Parenchymal Agents in Zone 1 (Figure 2) in the loss outcome. Macrophage Agents had significantly more contacts with Parenchymal Agents at 100 and 200 ticks in the loss vs. the win outcome (data not shown). The drop in percentage of engaged agents in the win outcomes for Macrophage and NK Agents indicated an increase in the number of agents present that were not engaged (Figure 3E and $3 \mathrm{~F}$ ). The engaged NK Agents in Zone 1 were killing the virally infected Parenchymal Agents or their "stressed" neighbors (additional files 6 and 9). The median number of contacts with Parenchymal agents per NK Agent was significantly greater for the loss vs. the win outcome at 100 and 200 ticks (data not shown), because there were more infected Parenchymal Agents (Figure 2). 
Specific interactions between agents in each zone were counted and the results from Zone 2 (the lymphoid tissue zone) are shown in Figure 5. The contact pairs in Figure 5 are listed with the agent recording the contact first. Since both agents would record a contact between them, the contacts are counted by two agent types in the figure. After arrival to present antigen in Zone 2, significantly higher frequencies of contacts per Dendritic Agent with the TCell and BCell Agents at these early time points were associated with the win outcome. No outcome-associated differences in the Dendritic Agents' median quantity of contacts with CTL Agents were found at 100 and 200 ticks (Figure 5).

In contrast, significantly fewer Dendritic Agent contacts were made per BCell Agent for the win vs. the loss outcomes at 100 and 200 ticks in Zone 2 (Figure 5). BCell Agents also made antibody after contact with virus [64,65], but BCell Agent contacts with free virus were not counted in these analyses. The effector TCell, CTL, and BCell Agents generated by this initial activity then migrated via Zone 3 (the blood) to Zone 1 to eliminate the virally infected Parenchymal Cells directly or via antibody production (additional files 24, 25, 26 and Figure 2).

The median number of contacts per TCell Agent with BCell and Dendritic Agents was not greater for the win outcome (Figure 5). However, the win outcomes were associated with far more TCell Agents making at least one specific contact with a BCell Agent (fourteen times more per simulation run by 100 ticks) or a Dendritic Agents (ten times more per simulation run by 100 ticks) than the loss outcomes in Zone 2 (data not shown). This reiterates the role for TCell Agent engagement for a win (Figure 3B).

The CTL Agents had a greater percentage of agents engaged in the loss outcome (Figure 3D), and more were present in Zone 1 (the functional tissue zone) in the loss outcome (additional file 26). The CTL Agents contacted Dendritic Agents in Zone 2, but there were no differences in the median number of these contacts per CTL Agent (Figure 5) in Zone 2 at 100 or 200 ticks for the win and loss outcomes.

The TCell and BCell Agents need contact with each other in Zone 2 for full activation and antibody production to occur (additional files 13 and 14; [40,52]). The median number of contacts per BCell Agent with TCell Agents was not different for the first 100 ticks of win vs. loss outcomes, but more TCell Agent contacts per BCell agent by 200 ticks was associated with the win outcome (Figure 5). In addition, there were seven times as many BCell Agents that made specific contact with TCell Agents in the same interval for wins vs. losses (data not shown). By 200 ticks, there were almost nine times as many BCell Agents that had made contact with a TCell Agent in Zone 2 for the win vs. the loss outcomes (data not shown).

\section{Interaction history for engaged agents over time}

The median number of contacts and the interquartile range ( \pm 25 th percentile) were plotted to describe the overall contact history at every 100 ticks of the simulation in all zones with all agent types (Figure 6A, B, C, D, E, and 6F).

The BCell Agents and TCell Agents had a higher percentage of agents engaged in the virtual immune response when there was a win outcome (Figure $3 \mathrm{~A}$ and $3 \mathrm{~B}$ ), but the median number of contacts or links per node were greater in the loss outcome throughout the time course (Figure 6A and 6B). Because the data presented in Figure 6 are cumulative, increases in the numbers of agents without contacts at the end of 
simulation runs resulted in decreases in the median links per node (Figure 6A, B, and $6 \mathrm{~F}$ ). For the TCell and BCell Agents (Figure 6A and 6B) these are memory cells (additional files 24 and 25). NK Agents (Figure 6F) are likely unable to make contacts (kill remaining infected Parenchymal Agents) because anti-inflammatory cytokines predominate (additional files 9 and 28). These are produced by Macrophage Agents of type 2, or anti-inflammatory Macrophage Agents ([62]; additional files 10 and 27).

In contrast to the BCell and TCell Agents, the CTL Agents and Natural Killer Agents had a higher percentage of agents engaged when there was a loss outcome (Figure 3), and CTL Agents also had more links per node for the loss outcome after 100 ticks (Figure 6D). NK Agents had higher median numbers of links per node at 100 and 200 ticks for the loss outcome (Figure 6F). The difference between cytotoxic T lymphocytes and natural killer cells is that the cytotoxic $\mathrm{T}$ lymphocytes require antigen presentation in the lymph node (to become activated to kill) but the natural killers cells do not $[66,67]$, so there was less delay in engagement and killing for the NK Agents. Although there were no significant differences in the number of contacts per CTL Agent at 100 ticks between the win and loss outcomes (Figure 6D), from 200 ticks onward the CTL Agents had more contacts per agent in the loss outcome.

The pattern observed for the Macrophage Agents was more agents engaged (Figure 3E) and more contacts (Figure 6E) in the loss outcomes than the win outcomes. As scavengers, the Macrophage Agents (Figure 6E) interacted with all agents, in most cases when the agents had died via apoptosis (Figure 1). Engaged Macrophage Agents had the most contacts per agent with Parenchymal Agents (specifically) compared to all other agents at 100 ticks, and significantly more in the loss outcomes (data not shown), perhaps because they contacted more infected and apoptotic Parenchymal Agents and then killed and/or phagocytosed them ([68-70]; additional file 10).

\section{The frequency distributions of the immune network contacts for the BIS_2010}

The frequency distributions of the meaningful contacts or links between the agents representing leukocytes (nodes) are shown in Figures 7 and 8, and additional files 31, 32, and 33. These plots are the logarithmically transformed, aggregate data from the win outcomes separated from the aggregate loss outcomes, with the cumulative numbers of links per node for data collected at 400 ticks and 1000 ticks (Figures 7 and 8, respectively). The median number of links per node is in brackets for the engaged agents in the aggregate data. These "time" points in the simulation run were chosen because at 400 ticks most of the agents' activities (contacts with other agents) had reached a level near the end-point value (Figure 6). The frequency distribution is a power law distribution in all cases except for the data from the Granulocyte Agents and the Natural Killer Agents. The functional relationship was tested on the raw data using a Spearman non-parametric test for correlation. Similar statistical results were obtained using linear regression on the log-transformed data (not shown). Remarkably similar results were obtained when other initial conditions were used, including 20, 100, and 300 Dendritic Agents, (1000 ticks shown in additional files 31, 32, and 33; statistics are summarized in additional file 34).

Thus the frequency distribution of the network interactions between immune cellagents during the simulated immune response is scale-free, suggesting that the network interactions of the immune system are as well. This result is not surprising for the 
system as a whole (Figure $7 \mathrm{H}$ and $7 \mathrm{P}$, and Figure $8 \mathrm{H}$ and $8 \mathrm{P}$ ) given that the interactions between elements in complex biological systems can generally be characterized as scale-free [7,9,71-74]. The frequency distributions for most of the different agent types were also scale-free, with the exceptions mentioned above, while the Granulocyte Agents did not have enough data points to test.

The agents representing granulocytes had the fewest agents engaged (data not shown). They also had the lowest median number of links (Figure 7G and 7O and Figure 8O). This is because granulocyte behavior, killing pathogens via release of reactive oxygen species and potent protease enzymes, is dependent upon activation by signals or cytokines/chemokines (additional file 21; [75]). Granulocytes have surface receptors to make physical contact with pathogens such as bacteria, parasites and fungi [76]. The Granulocyte Agent activation did not require receptor-mediated contact with other agents, but there were conditions where a few made contact with dying agents and necrotic debris, a condition thought to mimic pathogen contact (Figure 8G and 8O; [77]).

\section{Immune system hubs}

The Dendritic Agents had far more links than the other agents representing immune cells, making them hub agents of the virtual immune system (Table 1). Without representation of the necessity for antigen presentation to the adaptive immune cells, Figure 1 does not convey that Dendritic Agents are hubs. The analysis of the numbers of contacts between agents revealed this quality. By 200 ticks, a greater percentage of the Dendritic Agents were engaged compared to the other immune agents (Figure 3). As antigen presenting cells, dendritic cells may have more meaningful contacts than other immune cell types [78]. However, the median numbers of links for Dendritic Agents in the simulation seemed higher than expected at the end of the simulation runs and notably in the loss outcomes (Figure 6). This phenomenon was examined further (Table 2) to determine which agents the Dendritic Agents were interacting with at such high rates, and in which zone this was occurring.

\section{Dendritic Agent contacts with each agent type in Zones 1 and 2}

The striking difference in the number of contacts for Dendritic Agents between the win and loss outcomes was even more surprising when it was determined that most of the contacts were between Dendritic Agents themselves (Table 2). The Dendritic

Table 1 Median Number of Links for All Agents

\begin{tabular}{lllllllllll}
\hline Agent Type & $\mathbf{1 0}$ & $\mathbf{1 0}$ & \multicolumn{1}{c}{ Ticks } \\
\hline BCell Agents & $\mathbf{2 0 0}$ & $\mathbf{3 0 0}$ & $\mathbf{4 0 0}$ & $\mathbf{5 0 0}$ & $\mathbf{6 0 0}$ & $\mathbf{7 0 0}$ & $\mathbf{8 0 0}$ & $\mathbf{9 0 0}$ & $\mathbf{1 0 0 0}$ \\
\hline CTL Agents & 6 & 12 & 12 & 13 & 13 & 13 & 13 & 13 & 13 & 13 \\
\hline Dendritic Agents & 2 & 14 & 28 & 49 & 95 & 183 & 415 & 849 & 1495 & 2366 \\
\hline Granulocyte Agents & 0 & 0 & 1 & 1 & 1 & 1 & 1 & 1 & 1 & 1 \\
\hline Macrophage Agents & 2 & 2 & 2 & 2 & 2 & 2 & 2 & 2 & 2 & 2 \\
\hline Natural Killer Agents & 9 & 9 & 9 & 9 & 8 & 8 & 8 & 8 & 7 & 7 \\
\hline TCell Agents & 7 & 8 & 8 & 8 & 7 & 7 & 7 & 7 & 7 & 7 \\
\hline All & 3 & 6 & 6 & 6 & 6 & 6 & 7 & 8 & 9 & 11 \\
\hline
\end{tabular}

The median numbers of contacts for each agent type representing a leukocyte is given, including only the engaged agents. These are the contacts from all 146 simulation runs, including the win outcome and the loss outcomes combined. The results are shown for every 100 ticks of the simulation runs. 
Table 2 Dendritic Agent Median Contacts at 1000 ticks

\begin{tabular}{lllll}
\hline Outcome & WIN & & LOSS & \\
\hline Agent Type & Zone 1 & Zone 2 & Zone 1 & Zone 2 \\
\hline BCell Agents & 2 & 47 & 1 & 27 \\
\hline CTL Agents & 2 & 17 & 1 & 9 \\
\hline Dendritic Agents & 1 & 79 & 1 & 3085 \\
\hline Granulocyte Agents & 1 & 1 & 1 & 1 \\
\hline Macrophage Agents & 1 & 1 & 1 & 1 \\
\hline Natural Killer Agents & 1 & 1 & 1 & 1 \\
\hline TCell Agents & 1 & 10 & 1 & 5 \\
\hline
\end{tabular}

The median numbers of contacts that each engaged Dendritic Agent had with each agent type representing an immune cell type is given. The contacts are separated into columns of those accumulated in Zone 1 and then Zone 2, with the contacts from Zone 1 included with those in Zone 2 . The data from the simulation runs are also separated into columns by their outcome (wins, $\mathrm{n}=100$; loss, $\mathrm{n}=46$ ).

Agents interacted with other agents first in Zone 1 (tissue), and then in Zone 2 (lymph nodes; Figure 4). The data for the number of contacts made in Zone 2 includes the contacts from Zone 1 because the counting was not reset when the agents changed zones. Besides contacting themselves in Zone 2, the engaged Dendritic Agents actually had more contacts (higher medians) for their numbers of contacts with BCell, TCell and CTL Agents in the win outcomes than in the loss outcomes (Table 2). This models the antigen-presenting function of dendritic cells. The numerous contacts between Dendritic Agents at the end represented an emergent phenomenon for the simulation, with biological correlation discussed below.

\section{Discussion}

The value of applying network theory to understand relationships embedded in biological data is becoming better appreciated [74]. Defining the interactions between elements of biological systems as networks and characterizing the topology has broad application, from the study of protein-protein interactions in small organisms [7,79-82] to evolution of the proteins of the immune system [83] to defining networks of disease genotypes [73] and phenotypes $[13,84,85]$. The arbitrary definition of nodes and edges distinguishes all of the biological network analyses, and determines what information can be derived from the application of network theory to biological mechanisms.

Here we chose to define the network nodes as agents representing cells of the immune system, and edges as physical contacts made between agents representing immune cell types (via implied surface receptors) during a simulated immune response. Since an immune response to a pathogen is a dynamic process that involves sequential steps and movement of cells to different locations in the body to communicate information, the network defined above requires a dynamic analysis. This type of analysis cannot be conducted quantitatively in a living organism, but it can be conducted virtually using an agent-based model of the immune system.

The frequency distribution of the interactions between the agents representing immune cells was found to be scale-free for a range of starting conditions (Figure 8 and additional files 31,32, and 33), and the agents representing dendritic cells acted as hubs in the immune system network. This is consistent with our earlier results, using a simpler version of the simulation [9]. The new observations from this work include the analysis of the agent interactions recorded over virtual time, the individual agent 
types recording the types of agents they contacted, and in which zone the interactions were taking place.

One observation that arose from the assessment of the interactions between the immune cell agents was that the majority of agents present did not interact during the simulation (Figure 3). In general, the agents representing innate immune cells (Dendritic, Macrophage, and Natural Killer Agents) were more engaged at the beginning of the simulation (Figure 3C, E, and 3F; $[56,66,86,87]$ ). NK cells kill infected cells, but without the prior need for antigen presentation that adaptive immune cells require $[67,88,89]$. Macrophages innately recognize pathogens and infected, apoptotic or necrotic cells (additional files 10,11, and 12), but antibody attachment to infected cells or pathogens also helps macrophages find their targets [90]. The cells of the innate immune system, by definition, are ready to fight common pathogens at the first detection of "danger signals" [91-95]. In contrast, Granulocyte Agents had the least meaningful interactions of all of the agent types (Table 1) because they were programmed, like neutrophils, to become activated by signals or pathogens in the environment, rather than by specific interactions with other agents [75,76].

The overall lack of engagement for the other agent types is consistent with the real functioning of the immune system, validating the behavioral rules for the agents of the BIS_2010. Normal immune responses do not engage all of the cells of the immune system and in fact, biological conditions that involve overactive immune system engagement are septic shock [96] or systemic inflammatory response syndrome [77]. These conditions involve a systemic inflammatory response, with or without detectable infection, and a mortality rate near $50 \%$.

Our goal was to determine what characteristics of the immune system's communication metrics distinguished successful elimination of virus, or the win outcome, from the loss outcome. Both the numbers of agents making contacts (specific engagements) as well as the numbers of specific contacts per agent were compared. Communication between specific agents early in the simulation runs was found to be critical. Significantly more contacts between Dendritic Agents and both TCell and BCell Agents, occurring at 200 ticks or earlier, were associated with the win outcome (Figure 5). In Zone 2, far more TCell Agents contacted BCell Agents and Dendritic Agents in the first 100 ticks for the win outcomes (data not shown), but the median numbers of these specific contacts per TCell Agent did not differ (Figure 5). The same was true for the number of BCell Agents contacting TCell Agents. Additional files 24 and 25 show the effector and memory TCell and BCell Agents generated by the specific contacts that migrated to Zone 1 (via Zone 3). These results are consistent with the data in Figure 3 showing that only the BCell Agents and TCell Agents had more agents engaged during the simulation runs with the win outcome. The necessity for rapid communication, namely antigen presentation to lymphocyte agents, is valid simulation behavior for a win outcome [56].

CTL Agents also had to make specific contact with Dendritic Agents in Zone 2 (additional file 19) and migrate to Zone 1 to kill infected Parenchymal Agents (additional file 20) [97-99]. Unexpectedly, early in the simulation, the numbers of Dendritic Agent contacts with CTL Agents was not different (Figure 5). In the loss outcome, CTL Agents did not encounter Dendritic Agents and proliferate soon enough, so more Parenchymal Agents became infected (due to the continuous replication of virus) and 
significantly more CTL Agents became engaged. More effector CTL Agents made their way to Zone 1 (via Zone 3) in the loss outcome (additional file 26).

The most striking emergent outcome was the difference in the number of links per node in the win and loss outcomes for the Dendritic Agents (Figure 6), as well as the abundance of Dendritic Agents in Zone 2 in the loss outcome (Figure 4). The increase in the numbers of Dendritic Agents in Zone 2 at the end of the simulation runs with the loss outcomes is consistent with the high median numbers of contacts between these agents shown in Table 2, and the "lump" of points with more links per node in Figure $8 \mathrm{~K}$ at 1000 ticks, compared to Figure $7 \mathrm{~K}$ at 400 ticks. This outcome is validated by studies using in vivo using dual-laser microscopy [26]. Dendritic cells carry out tissue surveillance, phagocytose and process antigens, and present them to lymphocytes in the lymph node $[56,100,101]$. During inflammatory responses the lymph nodes become engorged with cells, and dendritic cells have a role in tissue remodeling of the lymph node to accommodate the influx [102]. They spread themselves along fibro-reticular networks in lymph nodes [103]. This requires "stepping" over other dendritic cells in the search for an open space on a fibroblast. As such, this migration process involves contacting other dendritic cells. The searching for an open space behavior was programmed into Dendritic Agents as normal behavior (additional file 8; [104]). The structural organization in lymph nodes enhances the ability of dendritic cells to probe incoming T-lymphocytes to find an antigen-matched lymphocyte [44,103]. T-lymphocytes travel from lymph node to lymph node [105], and must traverse the long processes of dendritic cells that are probing them [104], a process which adds to the contacts. The reason for the high numbers of contacts in the loss outcome for Dendritic Agents was the abundance of infected Parenchymal Agents in Zone 1, and the stimulation of more Dendritic Agents that traveled to Zone 2. This phenomenon is emergent, and only occurs in the loss outcome, validating the agent behavior rules in the BIS_2010.

As the previous model had shown [106], the Dendritic Agents represent the immune cells that are the hubs of the immune system. One could hypothesize that for preventing an undesired immune response such as transplant rejection, the best cellular target for therapy would be dendritic cells leaving the transplant tissue. Testing such a hypothesis would require a method for stopping the dendritic cells before they presented antigen in the lymph nodes. The dendritic cells also make signals that attract other leukocytes to the tissue, so such signals would have to be neutralized as well. This would be a difficult hypothesis to test, because both direct and indirect communication by the dendritic cells would have to be eliminated. In addition, only those dendritic cells presenting antigen from the graft should be targeted, to avoid diminishing the immune response to other pathogens.

In summary, we found that recording and analyzing direct interactions between agent types representing leukocytes using an agent-based model recapitulated what is believed to occur in vivo during an immune response. The results support the immuno-ecology idea that the more rapid the initial response, the better, despite the highly decentralized nature of the system's components. In addition, effectiveness is more important than efficiency for the immune system [1]. Agent-based modeling allows the analysis of the dynamic interactions between the elements of complex systems, a unique approach for biology. 


\section{Conclusions}

To gain insight into complex systems like the immune system, it is necessary to expand the types of approaches that we use to study them. An agent-based model of any complex system could be used to study its dynamic network interactions. Here we have used the agent-based modeling approach in combination with a dynamic network analysis to virtually observe what cannot be observed in vivo. All disease processes involve the immune system at some level. Any insight that can be gained through the application and combination of modeling and network analyses to the current body of knowledge of the immune system is valuable information. New techniques for analyzing collected scientific data in immunology are important for understanding disease processes and finding new ways to intervene.

\section{Availability and requirements}

The new version, BIS_2010 is available as a Java archive file (jar) at: http://digitalunion. osu.edu/r2/summer06/sass/download.html and as additional file 35. The BIS_2010.jar file must be downloaded as well as the RepastJ launcher (Repast_J_3.1_Installer.exe), available at: http://sourceforge.net/projects/repast/files/.

The source code was written in Java and compiled with Eclipse [107] using Java Runtime Environment 6, so a JRE version of at least 6.0 must be installed on the computer used to run RepastJ and the BIS_2010. Once the necessary software is on the computer, the RepastJ executable jar must be run as per the instructions included with the software. The Repast toolbar will appear and one must click on the folder on the left. A "Load Model" panel will appear, and in the "Demo Models" list one must scroll down and choose "Other Models", then "Add". The "Open" panel allows one to locate the BIS_2010.jar and "Open" it. Then "Load" must be chosen in the "Load Model" panel to make the Graphical User Interface (GUI) appear. In the "Parameters" menu at least three choices must be made. First, one must choose to set one of the challenges to the immune system at the top of the list. "Set_ViralInfection:" and "Set_Bacti:" are the verified choices. A ' 1 ' must be typed in to replace the ' 0 ' for one of the choices. Following that are "StopSimulationAt:" and "CountIncrement". If these are unchanged, the simulation will run for 1001 ticks and collect contact data every 100 ticks. One text file will be recorded with the quantities of all elements of the simulation at every tick if it reaches the "StopSimulationAt:" tick. Two text files will be recorded every time the "countIncrement" is passed. All of these files will appear in the Repast folder. To prevent many files from being generated, the "CountIncrement" can be set equal to "StopSimulationAt:". Any other parameters may be altered, and the simulation is started with the triangle in the toolbar. The simulation can be stopped with the square button in the toolbar.

The source code will only be made available through collaboration agreement with the contact author.

\section{Additional material}

Additional file 1: Table of agents, cells, signals, and soluble mediators. The BIS_2010 agents and signals and their corresponding cells and soluble mediators A table listing all of the elements in the simulation, with citations. Additional file 2: Table of citations for agent behaviors. Summary of citations for agent behaviors. A table listing the agents, their behaviors, and citations for the simulation rules regarding their behaviors.

Additional file 3: Zone 1: A generic tissue space. A screen-shot showing the appearance of the simulation when it runs. 
Additional file 4: A list of all parameters. Initial parameter values. A list of all of the parameters in the simulation, most of which are accessible from the GUI.

Additional file 5: Key to state diagrams. A key to the symbols and colors in the state diagrams.

Additional file 6: State diagram: Parenchymal Agents (PC), Zone 1. A state diagram of the potential PC behavioral sequences in Zone 1.

Additional file 7: State diagram: Dendritic Agents (DCs) Zone 1. A state diagram of the potential DC behavioral sequences in Zone 1 .

Additional file 8: State diagram: Dendritic Agents (DCs), Zone 2. A state diagram of the potential DC behavioral sequences in Zone 2 .

Additional file 9: State diagram: Natural Killer Agents (NKs) in Zone 1. A state diagram of the potential NK behavioral sequences in Zone 1.

Additional file 10: State diagram: Macrophage agents (MФs), Zone 1. A state diagram of the potential MФ behavioral sequences in Zone 1.

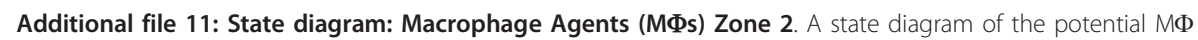
behavioral sequences in Zone 2 .

Additional file 12: State diagram: Macrophage Agents (MФs), Zone 3. A state diagram of the potential MФ behavioral sequences in Zone 3.

Additional file 13: State diagram: BCell Agents (Bs) in Zone 2 (Part 1). A state diagram of the potential B behavioral sequences in Zone 2 .

Additional file 14: State diagram: BCell Agents (Bs) in Zone 2 (Part 2). A state diagram of the potential B behavioral sequences in Zone 2 .

Additional file 15: State diagram: BCell Agents (Bs) in Zones 3 and 1. A state diagram of the potential B behavioral sequences in Zones 3 and 1 .

Additional file 16: State diagram: TCell Agents (Ts) in Zone 2 (Part 1). A state diagram of the potential T behavioral sequences in Zone 2 .

Additional file 17: State diagram: TCell Agents (Ts) in Zone 2 (Part 2). A state diagram of the potential T behavioral sequences in Zone 2

Additional file 18: State diagram: T Cell agents (Ts) in Zone 1. A state diagram of the potential T behavioral sequences in Zone 1.

Additional file 19: State diagram: Cytotoxic T Lymphocyte Agents (CTLs) in Zones 2 and 3. A state diagram of the potential CTL behavioral sequences in Zones 2 and 3.

Additional file 20: State diagram: Cytotoxic T Lymphocyte Agents (CTLs) in Zone 1. A state diagram of the potential CTL behavioral sequences in Zone 1.

Additional file 21: State diagram: Granulocyte Agents (GRAN) in Zones 3, 1. A state diagram of the potential GRAN behavioral sequences in Zones 3 and 1.

Additional file 22: State diagram: Portal Agents (Portals). A state diagram describing the Portals.

Additional file 23: Percentage of win or loss outcomes and ticks to eliminate infected agents for different starting conditions. A figure showing the outcomes when the initial number of Dendritic Agents is varied.

Additional file 24: The number of Effector and Memory TCell Agents in Zone 1 for the duration of the simulation for the win and loss outcomes. A figure that shows the average numbers of TCell Agents in Zone 1 for the duration of the simulation.

Additional file 25: The number of Effector and Memory BCell Agents in Zone 1 for the duration of the simulation for the win and loss outcomes. A figure that shows the average numbers of Effector and Memory BCell Agents in Zone 1 for the duration of the simulation.

Additional file 26: The number of Effector and Memory CTL Agents in Zone 1 for the duration of the simulation for the win and loss outcomes. A figure that shows the average numbers of Effector and Memory CTL Agents in Zone 1 for the duration of the simulation.

Additional file 27: The number of Macrophage Agents in Zone 1 for the duration of the simulation for the win and loss outcomes. A figure that shows the average numbers of Macrophage Agents in Zone 1 for the duration of the simulation.

Additional file 28: The number of Natural Killer Agents in Zone 1 for the duration of the simulation for the win and loss outcomes. A figure that shows the average numbers of Natural Killer Agents in Zone 1 for the duration of the simulation.

Additional file 29: The number of Granulocyte Agents in Zone 1 for the duration of the simulation for the win and loss outcomes. A figure that shows the average numbers of Granulocyte Agents in Zone 1 for the duration of the simulation.

Additional file 30: Additional file references. A list of references cited in all of the additional files.

Additional file 31: Frequency distributions of contacts for each immune agent type at 1000 ticks with a starting condition of $\mathbf{2 0}$ Dendritic Agents. A figure that shows the frequency distribution of contacts for each immune agent type at the end of the simulation (120 simulation runs combined; 20 Dendritic Agents starting condition) with the win and loss outcomes separated. 

starting condition of 100 Dendritic Agents. A figure that shows the frequency distribution of contacts for each immune agent type at the end of the simulation (120 simulation runs combined; 100 Dendritic Agents starting condition) with the win and loss outcomes separated.

Additional file 33: Frequency distributions of contacts for each immune agent type at 1000 ticks with a starting condition of $\mathbf{3 0 0}$ Dendritic Agents. A figure that shows the frequency distribution of contacts for each immune agent type at the end of the simulation (120 simulation runs combined; 300 Dendritic Agents starting condition) with the win and loss outcomes separated.

Additional file 34: Table of statistics for Figure 8 and additional files 31, 32, and 33. A table of statistics including the number of data points, Spearman $r$ value for correlation and the $p$-value for the correlation statistic for all of the frequency distribution diagrams for each agent shown in Figure 8 and additional files 31, 32, and 33.

Additional file 35: An executable jar file for the simulation, BIS_2010.

\section{List of Abbreviations and Definitions}

Agent: the interactive entities of the model with rules for behavior; behavior: what the agents are programmed to do, listed in additional file 2; BIS: Basic Immune Simulator; degree: the number of edges or links a node has with other nodes; edge: a connection between two nodes, the same as a link; emergent behavior: behavior that occurs as an unforeseen consequence of the combinations of rules from other agents; engaged: an agent that has made one or more contacts, hub: a node in a network that has the most edges or links; imposed behavior: intentionally programmed behavior (behavior necessary for a successful immune response); leukocyte: a white blood cell, all leukocytes are part of the immune system; link: a connection between two nodes, the same as an edge; Moore neighborhood: the eight adjacent locations to a central location in a rectangular matrix; node connectivity: the number of edges or links a node has with other nodes; power-law distribution: a frequency distribution that fits the funcion $y=\alpha x^{\beta}$; scale-free distribution: a frequency distribution that spans several powers of a base number; signal: a representation of diffusing substances produced by the agents; state diagram: a diagram (directed graph) of a finite state automaton; stigmergy: the indirect communication between agents allowed by changes to the environment such as signal production; tick: a discrete time step in the simulation; zone: a virtual environment where agents and signals interact.

\section{Competing interests}

The authors declare that they have no competing interests.

\section{Authors' contributions}

VAF created, updated and modified the BIS_2010 to collect data for network analysis. She also conceived of the AgentVerifier, and modified the BIS_2010 to produce output for the AgentVerifier created by CE, JD, SM, BB and MK. VAF wrote the initial draft of the manuscript. GB and CBM made substantive intellectual contributions to the manuscript and interpretation of data, as well as the drafting and revision of the manuscript. SM, JD, CE, BB and MK designed and created specialized software for data analysis including and in addition to the AgentVerifier. CE, JD and LD were involved in drafting parts of the manuscript. All authors were involved in revising the manuscript critically for intellectual content, and have given their final approval for the version to be published.

\section{Acknowledgements}

The project described was supported by award number R21HL093675 and R01HL067176 from the National Heart, Lung, and Blood Institute of the National Institutes of Health. The content is solely the responsibility of the authors and does not necessarily represent the official views of the National Heart, Lung, and Blood Institute or the National Institutes of Health.

Although the BIS_2010 runs on a personal computer, the IBM Opteron Cluster of the Ohio Supercomputer Center in Columbus, Ohio [108] was used to perform the batch runs of the simulation.

\section{Author details}

'Department of Internal Medicine, Division of Pulmonary, Allergy, Critical Care and Sleep Medicine, The Ohio State University Medical Center, Davis Heart and Lung Research Institute, Columbus, OH, USA. ${ }^{2}$ Department of Medicine, University of Alberta, Edmonton, Alberta, Canada. ${ }^{3}$ Department of Computer Science and Engineering, The Ohio State University, Columbus, OH, USA. ${ }^{4}$ Department of Medicine Administration, The Ohio State University Medical Center, Columbus, OH, USA.

Received: 27 August 2010 Accepted: 19 January 2011 Published: 19 January 2011

\section{References}

1. Orosz CG: An introduction to immuno-ecology and immuno-informatics. In Design Principles for the Immune System and Other Distributed Autonomous Systems. Edited by: Segel L, Cohen IR. New York: Oxford University Press; 2001:

2. Fadok VA, Bratton DL, Konowal A, Freed PW, Westcott JY, Henson PM: Macrophages that have ingested apoptotic cells in vitro inhibit proinflammatory cytokine production through autocrine/paracrine mechanisms involving TGFB, PGE2, and PAF. Journal of Clinical Investigation 1998, 101:890-898.

3. Haslett C: Granulocyte apoptosis and its role in the resolution and control of lung inflammation. American Journal of Respiratory and Critical Care Medicine 1999, 160:S5-S11. 
4. Rydell-Tormanen $K$, Uller L, Erjefalt JS: Direct evidence of secondary necrosis of neutrophils during intense lung inflammation. European Respiratory Journal 2006, 28:268-274.

5. Yamasaki S, Ishikawa E, Sakuma M, Hara H, Ogata K, Saito T: Mincle is an ITAM-coupled activating receptor that senses damaged cells. Nature Immunology 2008, 9:1179-1188.

6. Barabasi A-L, Albert R: Emergence of Scaling in Random Networks. Science 1999, 286:509-512.

7. Jeong H, Tombor B, Albert R, Oltvai ZN, Barabasi A-L: The large-scale organization of metabolic networks. Nature 2000, 407:651-654.

8. Albert R, Jeong H, Barabasi A-L: Error and attack tolerance of complex networks. Nature 2000, 406:378-382.

9. Folcik VA, Orosz CG: An Agent-Based Model Demonstrates that the Immune System Behaves Like a Complex System and a Scale-Free Network. Tenth Annual Swarm Agent-Based Simulation Meeting; Notre Dame University, Indiana, USA 2006 [http://www.nd.edu/ swarm06/Schedule/schedule.html].

10. Tieri P, Valensin S, Franceschi C, Morandi C, Castellani GC: Memory and Selectivity in Evolving Scale-Free Immune Networks Berlin Heidelberg: Springer-Verlag; 2003.

11. Frankenstein Z, Alon U, Cohen IR: The immune-body cytokine network defines a social architecture of cell interactions. Biology Direct 2006, 1:32.

12. Tieri P, Valensin S, Latora V, Castellani GC, Marchiori M, Remondini D, Franceschi C: Quantifying the relevance of different mediators in the human immune cell network. Bioinformatics 2005, 21:1639-1643.

13. Fuite J, Vernon SD, Broderick G: Neuroendocrine and immune network re-modeling in chronic fatigue syndrome: An exploratory analysis. Genomics 2008, 92:393-399.

14. Grasse P-P: La reconstruction du nid et les coordinations inter-individuelles chez Bellicositermes Natalensis et Cubitermes sp. La theoriede la stigmergie: Essai d'interpretation du comportement des Termites constructeurs. Insect Sociology 1959, 6:41-80.

15. Bonabeau E, Dorigo M, Theraulaz G: Swarm Intelligence. From Natural to Artificial Systems New York, New York: Oxford University Press; 1999.

16. Folcik VA, An GC, Orosz CG: The Basic Immune Simulator: An Agent- Based Model to study the interactions between innate and adaptive immunity. Theoretical Biology and Medical Modelling 2007, 4:39.

17. Repast. Recursive Porous Agent Simulation Toolkit. [http://repast.sourceforge.net/repast_3/index.html].

18. Roitt I, Brostoff J, Male D: Immunology. 6 edition. London: Mosby, Harcourt Publishers Ltd.; 2001.

19. Delves PJ, Martin SJ, Burton DR, Roitt IM: Roitt's Essential Immunology. Eleventh edition. Malden, MA: Blackwell Publishing Ltd.; 2006.

20. Mestecky J, Ogra P, McGhee J, Lambrecht BN, Strober W, Eds: Mucosal Immunology. Boston: Elsevier Academic Press; Third 2005.

21. Armstrong JD, Gluck EH, Crapo RO, Jones HA, Hughes JMB: Lung tissue volume estimated by simultaneous radiographic and helium dilution methods. Thorax 1982, 37:676-679.

22. Quint LE, Glazer GM, Orringer MB, Francis IR, Bookstein FL: Mediastinal lymph node detection and sizing at $C T$ and autopsy. American Journal of Radiology 1986, 147:469-472.

23. Rusch WW, Asamura H, Watanabe H, Giroux DJ, Rami-Porta R, Golstraw P: The IASLC Lung Cancer Staging Project: A proposal for a new international lymph node map in the forthcoming seventh edition of the TNM classification for lung cancer. Journal of Thoracic Oncology 2009, 4:568-577.

24. Bogle G, Dunbar PR: T cell responses in lymph nodes. Wiley Interdisciplinary Reviews: Systems Biology and Medicine 2009.

25. Cahalan MD, Gutman GA: The sense of place in the immune system. Nature Immunology 2006, 7:329-332.

26. Germain RN, Bajenoff M, Castellino F, Chieppa M, Egen JG, Huang AYC, Ishii M, Koo LY, Qi H: Making friends in out-ofthe-way places: how cells of the immune system get together and how they conduct their business as revealed by intravital imaging. Immunological Reviews 2008, 221:163-181.

27. An G, Lee IA: Complexity, emergence and pathophysiology: Using agent based computer simulation to characterize the non-adaptive inflammatory response. InterJournal Complex Systems 2000, Manuscript\#[344].

28. An G: Complexity in ICU. Concepts for developing a collaborative in silico model of the acute inflammatory response using agent-based modeling. Journal of Critical Care 2006, 21:105-110.

29. An G: Introduction of an agent-based multi-scale modular architecture for dynamic knowledge representation of acute inflammation. Theoretical Biology and Medical Modelling 2008, 5:11.

30. North MJ, Collier NT, Vos JR: Experiences Creating Three Implementations of the Repast Agent Modeling Toolkit. ACM Transactions on Modeling and Computer Simulation 2006, 16:1-25.

31. Langrish CL, Chen Y, Blumenschein WM, Mattson J, Basham B, Sedgwick JD, McClanahan T, Kastelein RA, Cua DJ: IL-23 drives a pathogenic $T$ cell population that induces autoimmune inflammation. The Journal of Experimental Medicine 2005, 201:233-240.

32. Harrington LE, Hatton RD, Mangan PR, Turner H, Murphy TL, Murphy KM, Weaver CT: Interleukin 17-producing CD4+ effector T cells develop via a lineage distinct from the T helper type 1 and 2 lineages. Nature Immunology 2005, 6:1123-1132.

33. Park H, Li Z, Yang XO, Chang SH, Nurieva R, Wang Y-H, Wang Y, Hood L, Zhu Z, Tian Q, Dong C: A distinct lineage of CD4 T cells regulates tissue inflammation by producing interleukin 17. Nature Immunology 2005, 6:1133-1141.

34. Mangan PR, Harrington LE, O'Quinn DB, Helms WS, Bullard DC, Elson CO, Hatton RD, Wahl SM, Schoeb TR, Weaver CT: Transforming growth factor-B induces development of the Th17 lineage. Nature 2006, 441:231-234.

35. Hori S, Sakaguchi S: Foxp3: a critical regulator of the development and function of regulatory T cells. Microbes and Infection 2004, 6:745-751.

36. Bacchetta R, Gregori S, Roncarolo M-G: CD4+ regulatory T cells: Mechanisms of induction and effector function. Autoimmunity Reviews 2005, 4:491-496.

37. Bettelli E, Carrier Y, Gao W, Korn T, Strom TB, Oukka M, Weiner HL, Kuchroo VK: Reciprocal developmental pathways for the generation of pathogenic effector $T_{H} 17$ and regulatory $T$ cells. Nature 2006, 441:235-238.

38. Quintana FJ, Basso AS, Iglesias AH, Korn T, Farez MF, Bettelli E, Caccamo M, Oukka M, Weiner HL: Control of Treg and Th17 cell differentiation by the aryl hydrocarbon receptor. Nature 2008, 453:65-72.

39. Feuerer M, hill JA, Mathis D, Benoist C: Foxp3+ regulatory T cells: differentiation, specification, subphenotypes. Nature Immunology 2009, 10:689-695. 
40. Chtanova T, Tangye SG, Newton R, Frank N, Hodge MR, Rolph MS, Mackay CR: T follicular helper cells express a distinctive transriptional profile, reflecting their role as non-Th1/Th2 effector cells that provide help for B cells. The Journal of Immunology 2004, 173:68-78.

41. Bryant VL, Ma CS, Avery DT, Li Y, Good KL, Corcoran LM, deWaalMalefyt R, Tangye SG: Cytokine-mediated regulation of human B cell differentiation into Ig-secreting cells: Predominant role of IL-21 produced by CXCR5+ T follicular helper cells. The Journal of Immunology 2007, 179:8180-8190.

42. Law AM: Simulation Modeling and Analysis. 4 edition. Boston: McGraw-Hill; 2007.

43. Ekbote C, Doolittle J, Block B, Marsh C, Folcik VA: Verification of an agent-based model: Meeting the challenge of verifying object-oriented code that executes stochastically. Swarmfest 2010; Santa Fe Complex, Santa Fe, New Mexico 2010 [http://www.swarm.org/index.php/Verification_of_an_Agent-Based_Model: _Meeting_the_Challenge_of_Verifying_Object-Oriented_Code_that_Executes_Stochastically].

44. Bajénoff $M$, Granjeaud $S$, Guerder $S$ : The strategy of $T$ cell antigen-presenting cell encounter in antigen-draining lymph nodes revealed by imaging of initial T cell activation. J Exp Med 2003, 198:715-724.

45. Miller MJ, Hejazi AS, Wei SH, Cahalan MD, Parker I: T cell repertoire scanning is promoted by dynamic dendritic cell behavior and random T cell motility in the lymph node. Proc Natl Acad Sci 2004, 101:998-1003.

46. Ingulli E, Mondino A, Khoruts A, Jenkins MK: In vivo detection of dendritic cell antigen presentation to CD4+ T cells. Journal of Experimental Medicine 1997, 185:2133-2141.

47. Garside P, Ingulli E, Merica RR, Johnson JG, Noelle RJ, Jenkins MK: Visualization of specific B and T lymphocyte interactions in the lymph node. Science 1998, 281:96-99.

48. Miga AJ, Masters SR, Durell BG, Gonzales M, Jenkins MK, Maliszewski C, Kikutani H, Wade WF, Noelle RJ: Dendritic Cell longevity and T cell persistence is controlled by CD154-CD40 interactions. Eur J Immunol 2001, 31:959-965.

49. Bousso P, Robey E: Dynamics of CD8+ T cell priming by dendritic cells in intact lymph nodes. Nature Immunology 2003, 4:579-585.

50. Crotty S, Felgner P, Davies H, Glidewell J, Villarreal L, Ahmed R: Cutting edge: Long-term B cell memory in humans after smallpox vaccination. The Journal of Immunology 2003, 171:4969-4973.

51. Mempel TR, Henrickson SE, vonAndrian UH: T-cell priming by dendritic cells in lymph nodes occurs in three distinct phases. Nature 2004, 427:154-159.

52. Okada T, Miller MJ, Parker I, Krummel MF, Neighbors M, Hartley SB, O'Garra A, Cahalan MD, Cyster JG: Antigen-engaged B cells undergo chemotaxis toward the T zone and form motile conjugates with helper T cells. PLoS Biology 2005, 3:e150.

53. Germain RN, Miller MJ, Dustin ML, Nussenzweig MC: Dynamic imaging of the immune system: progress, pitfalls and promise. Nature Reviews Immunology 2006, 6:497-507.

54. Oppmann B, Lesley R, Blom B, Timans JC, Xu Y, Hunte B, Vega F, Yu N, Wang J, Singh K, Zonin F, Vaisberg E, Churakova T, Liu M, Gorman D, Wagner J, Zurawski S, Liu Y-J, Abrams JS, Moore KW, Rennick D, de Waal-Malefyt R, Hannum C, Bazan JF, Kastelein RA: Novel p19 protein engages IL-12p40 to form a cytokine, IL-23, with biological activities similar as well as distinct from IL-12. Immunity 2000, 13:715-725.

55. Koch F, Stanzl U, Jennewein P, Janke K, Heufler C, Kampgen E, Romani N, Schuler G: High level IL-12 production by murine dendritic cells: Upregulation via MHC Class II and CD40 molecules and downregulation by IL-4 and IL-10. Journal of Experimental Medicine 1996, 184:741-746.

56. Hart DNJ: Dendritic Cells: Unique leukocyte populations which control the primary immune response. Blood 1997, 90:3245-3287.

57. Vieira PL, de Jong EC, Wierenga EA, Kapsenberg ML, Kalinski P: Development of Th1-inducing capacity in myeloid dendritic cells requires environmental instruction. J Immunol 2000, 164:4507-4512.

58. Moser M, Murphy KM: Dendritic cell regulation of $\mathrm{T}_{\mathrm{H}} 1-\mathrm{T}_{\mathrm{H}} 2$ development. Nature Immunology 2000, 1:199-205.

59. Tanaka H, Demeure CE, Rubio M, Delespesse G, Sarfati M: Human monocyte-derived dendritic cells induce naïve T cell differentiation into T helper cell type 2 (Th2) or Th1/Th2 effectors: Role of stimulator/responder ratio. J Exp Med 2000, 192:405-411.

60. Anderson CF, Lucas M, Gutierrez-Kobeh L, Field AE, Mosser DM: T cell biasing by activated dendritic cells. J Immunol 2004, 173:955-961.

61. Boniface K, Blom B, Liu Y-J, deWaal-Malefyt R: From interleukin-23 to T-helper 17 cells: human T-helper cell differentiation revisited. Immunological Reviews 2008, 226:132-146.

62. Couper KN, Blount DG, Riley EM: IL-10: The master regulator of immunity to infection. The Journal of Immunology 2008, 180:5771-5777.

63. Dubois B, Bridon J-M, Fayette J, Barthelemy C, Banchereau J, Caux C, Briere F: Dendritic cells directly modulate B cell growth and differentiation. Journal of Leukocyte Biology 1999, 66:224-230.

64. Sixt M, Kanazawa N, Selg M, Samson T, Roos G, Reinhardt DP, Pabst R, Lutz MB, Sorokin L: The conduit system transports soluble antigens from the afferent lymph to resident dendritic cells in the T cell area of the lymph node. Immunity 2005, 22:19-29.

65. Lopes-Carvalho T, Foote J, Kearney JF: Marginal zone B cells in lymphocyte activation and regulation. Current Opinion in Immunology 2005, 17:244-250.

66. Salazar-Mather TP, Orange JS, Biron CA: Early murine cytomegalovirus (MCMV) infection induces liver Natural Killer (NK) cell inflammation and protection through Macrophage Inflammatory Protein 1a (MIP-1a)-dependent pathways. J Exp Med 1998, 187:1-14.

67. Smyth MJ, Cretney E, Kelly JM, Westwood JA, Street SEA, Yagita H, Takeda K, van Dommelen SLH, Degli-Esposti MA, Hayakawa Y: Activation of NK cell cytotoxicity. Molecular Immunology 2005, 42:501-510.

68. Ricevuti G: Host tissue damage by phagocytes. Annals of the New York Academy of Sciences 1997, 832:426-448

69. Huynh M-LN, Fadok VA, Henson PM: Phosphatidylserine-dependent ingestion of apoptotic cells promotes TGF- $\beta 1$ secretion and the resolution of inflammation. The Journal of Clinical Investigation 2002, 109:41-50.

70. Anderson CF, Mosser DM: Cutting edge: Biasing immune responses by directing antigen to macrophage $\mathrm{Fcg}$ receptors. Journal of Immunology 2002, 168:3697-3701.

71. Barabasi A-L, Oltvai ZN: Network biology: Understanding the cell's functional organization. Nature Reviews Genetics 2004, 5:101-113. 
72. Barabasi A-L, Bonabeau E: Scale-Free Networks. Scientific American 2003, May:60-69.

73. Barabasi A-L: Network medicine-from obesity to the "diseasome". The New England Journal of Medicine 2007, 357:404-407.

74. Barabasi A-L: Scale-free networks: a decade and beyond. Science 2009, 325:412-413.

75. Segal AW: How neutrophils kill microbes. Annu Rev Immunol 2005, 23:197-223.

76. Parker LC, Whyte MKB, Dower SK, Sabroe I: The expression and roles of Toll-like receptors in the biology of the human neutrophil. Journal of Leukocyte Biology 2005, 77:886-892.

77. Zhang Q, Raoof M, Chen Y, Sumi Y, Sursal T, Junger W, Brohi K, Itagaki K, Hauser CJ: Circulating mitochondrial DAMPs cause inflammatory responses to injury. Nature 2010, 464:104-107.

78. Beltman JB, Maree AFM, Lynch JN, Miller MJ, deBoer RJ: Lymph node topology dictates T cell migration behavior. The Journal of Experimental Medicine 2007, 204:771-780

79. Lee I, Date SV, Adai AT, Marcotte EM: A probabilistic functional network of yeast genes. Science 2004, 306:1555-1558.

80. Almaas E, Oltvai ZN, Barabasi A-L: The activity reaction core and plasticity of metabolic networks. PLoS Computational Biology 2005, 1:e68.

81. Zotenko E, Mestre J, O'Leary DP, Przytycka TM: Why do hubs in the yeast protein interaction network tend to be essential: reexamining the connection between the network topology and essentiality. PLoS Computational Biology 2008, 4:1-16

82. He X, Zhang J: Why do hubs tend to be essential in protein networks? PLoS Genetics 2006, 2:0826-0834.

83. Ortutay $C$, Vihinen M: Efficiency of the immunome protein interaction network increases during evolution. Immunome Research 2008, 4:4

84. Goh K-I, Cusick ME, Valle D, Childs B, Vidal M, Barabasi A-L: The human disease network. Proceedings of the National Academy of Sciences 2007, 104:8685-8690.

85. Hidalgo CA, Blumm N, Barabasi A-L, Christakis N: A dynamic network approach for the study of human phenotypes. PLoS Computational Biology 2009, 5:e1000353.

86. Lodoen MB, Lanier LL: Natural killer cells as an initial defense against pathogens. Current Opinion in Immunology 2006, 18:391-398

87. Takeguchi O, Akira S: Innate immunity to virus infection. Immunological Reviews 2009, 227:75-86.

88. Beilhack A, Rockson SG: Immune traffic: A functional overview. Lymphatic Research and Biology 2003, 1:219-234.

89. Stetson DB, Mohrs M, Reinhardt RL, Baron JL, Wang Z-E, Gapin L, Kronenberg M, Locksley RM: Constitutive cytokine mRNAs mark Natural Killer (NK) and NK T Cells poised for rapid effector function. J Exp Med 2003, 198:1069-1076.

90. Casadevall A, Pirofski L: Antibody-mediated regulation of cellular immunity and the inflammatory response. TRENDS in Immunology 2003, 24:474-478.

91. Gallucci S, Lolkema M, Matzinger P: Natural Adjuvants: Endogenous activators of dendritic cells. Nature Medicine 1999, 5:1249-1255.

92. Srivastava P: Roles of heat shock protein in innate and adaptive immunity. Nature Reviews Immunology 2002, 2:185-194.

93. Shi Y, JE E, Rock KL: Molecular identification of a danger signal that alerts the immune system to dying cells. Nature 2003, 425:516-521.

94. Sansonetti PJ: The innate signaling of dangers and the dangers of innate signaling. Nature Immunology 2006, 7:1237-1242.

95. Matzinger P: Friendly and dangerous signals: is the tissue in control? Nature Immunology 2007, 8:11-13

96. Nguyen HB, Rivers EP, Abrahamian FM, Moran GJ, Abraham E, Trzeclak S, Huang DT, Osborn T, Stevens D, Talan DA, EDSEPSIS Working Group: Severe sepsis and septic shock: Review of the literature and emergency department management guidelines. Annals of Emergency Medicine 2006, 48:28-54.

97. Ridge JP, Rosa FD, Matzinger P: A conditioned dendritic cell can be a temporal bridge between a CD4+ T-helper and a T-killer cell. Nature 1998, 393:474-478.

98. Bennett SRM, Carbone FR, Karamalis F, Flavell RA, Miller JFAP, Heath WR: Help for cytotoxic-T-cell responses is mediated by CD40 signalling. Nature 1998, 393:478-480.

99. Schoenberger SP, Toes REM, vanderVoort EIH, Offringa R, Melief CJM: T-cell help for cytotoxic T lymphocytes is mediated by CD40-CD40L interactions. Nature 1998, 393:480-483.

100. Banchereau J, Steinman RM: Dendritic cells and the control of immunity. Nature 1998, 392:245-252.

101. Mellman I, Steinman RM: Dendritic cells: Specialized and regulated antigen processing machines. Cell 2001, 106:255-258

102. Webster B, Ekland EH, Agle LM, Chyou S, Ruggieri R, Lu TT: Regulation of lymph node vascular growth by dendritic cells. The Journal of Experimental Medicine 2006, 203:1903-1913.

103. Katakai T, Hara T, Lee J-H, Gonda H, Sugai M, Shimizu A: A novel reticular stromal structure in lymph node cortex: an immuno-platform for interactions among dendritic cells, T cells and B cells. International Immunology 2004, 16:1133-1142.

104. Lindquist RL, Shakhar G, Dudziak D, Wardemann H, Eisenreich T, Dustin ML, Nussenzweig MC: Visualizing dendritic cell networks in vivo. Nature Immunology 2004, 5:1243-1250.

105. Miller MJ, Wei SH, Cahalan MD, Parker I: Autonomous T cell trafficking examined in vivo with intravital two-photon microscopy. Proceedings of the National Academy of Sciences 2003, 100:2604-2609.

106. Folcik VA, Orosz CG: An Agent-Based Model Demonstrates that the Immune System Behaves Like a Complex System and a Scale-Free Network. Swarmfest 2006, Tenth Annual Swarm Agent-Based Simulation Meeting Notre Dame University, Indiana, USA:[http://www.nd.edu/ swarm06/Schedule/schedule.html].

107. Eclipse. [http://www.eclipse.org].

108. Ohio Supercomputing Center. [http://www.osc.edu].

doi:10.1186/1742-4682-8-1

Cite this article as: Folcik et al:: Using an agent-based model to analyze the dynamic communication network of the immune response. Theoretical Biology and Medical Modelling 2011 8:1. 